\title{
New Water Quality Model using Smart Decision Maker and Internet of Things for the Determination of Water Quality of River Shatt Al-Arab in Iraq
}

\author{
Ali Hadi Abdulwahid \\ Engineering Technical College, Southern Technical University, Basrah, Iraq
}

\begin{abstract}
Water pollution has become a major problem which needs to be addressed. Water quality is monitored by going to the water bodies and collecting water samples which are then sent for testing in laboratories. This is a time-consuming process and an ineffective method plus it's separate. In order to automate this process, we verified the water quality remotely. This was done with the help of sensors, transceiver modules and microcontroller Arduino Model. The system used three sensors: a pH sensor (SKU SEN0161), turbidity sensor (SEN0244) and a temperature sensor (DS18B20). All data collected were wirelessly transmitted to smartphones. In order to facilitate this transmission, we used the WiFi transmitter of which module was connected to Arduino. These sensors were deployed in the water bodies which were connected to a microcontroller. The data collected from the sensors were sent from the transmitter through the receiver to the smartphone.
\end{abstract}

Key words: Water quality model, smart decision maker, internet of things, water quality sensors, River Shatt Al-Arab, turbidity sensor

\section{INTRODUCTION}

Water contamination has become an important issue now a days. One of the main obstacles in this process is the current water quality detection system which is tested by going to water bodies, collecting samples and returning them to laboratories. These samples are then tested for impurities and contaminants. After that, a report is published about all the water bodies. This is an intermittent and time-consuming process as is the case after taking a sample of one aquatic body, the following sample is taken to the same aquatic body over a long period. During this time, the level of contamination may rise and become harmful. If detected early, it is possible to be treated at an early stage (Anuradha et al., 2018).

The proposed system provides the Water Quality (WQ) monitoring remotely and gives real-time updates. We have deployed three sensors in the water, namely, $\mathrm{pH}$ sensor, turbidity sensor and temperature sensor. These sensors collect data which are transferred to the smartphone application. This intelligent application displays real-time values for all sensors and uploads data to the ThinkSpeak channel. It also sends an SMS to the relevant authorities if the sensor readings are out of bounds (Bhardwaj, 2011).

Literature review: The rapid growth of the population stems from the depletion of available water resources and the decline in water quality. In addition, the quality of groundwater has been infected with herbicides and as a result of the accumulation of remnants of war. Rivers in Iraq are polluted by industrial waste and untreated wastewater (Annymous, 2019).

Ye et al. (2002) measured approximately describing the connection of sensors and environmental monitoring applications and refer mainly to the minimum energy consumption in wireless network applications. Papageorgiou (2003) analyzed different wireless modes, configurations and networks. He also analyzed protocols and layers in a wireless network while Tuan et al. gave a brief description of the characteristics and sensor design (Le Dinh et al., 2007). Qiao and Song (2010) introduced the quality monitoring system based on GPRS/GSM. The module collects the data and sends it to the monitoring center via. GPRS. It is an artificial method of data collection and other processes are slowly completed Qiao and Song (2010) while Amruta and Satish (2013) used the Base Station (BS) to collect information from distant remote sensors. The BS associated with the ZigBee module was powered by a skirting board in sunlight as a source of energy.

Liang et al. in the new arrival and evolution of the internet have been clarified to use the internet of things and the different techniques have been explained (Wang et al., 2015).

Corresponding Author: Ali Hadi Abdulwahid, College of Electrical and Electronic, Huazhong University of Science and Technology, Hubei, China 
While Barabde and Danve (2015) used the system to determine the physicochemical factors of water quality such as temperature, $\mathrm{pH}$, conductivity and the oxidation-reduction potential by ZigBee, Barabde and Danve (2015), Pavana and Padma (2016) studied water quality factors through Wireless composite Sensor Networks (WSN) and used the Raspberry Pi module with the Linux version (Pavana and Padma, 2016).

Problem statement: More likely to become regular monitoring in real time of the quality of drinking water is an essential feature of urban life for the possibility of natural or deliberate pollution. Among the different criteria used to measure the quality of water, the $\mathrm{pH}$ is the standard of evaluation which is particularly important. In order to be compatible with the World Health Organization (WHO), for example, a generally acceptable alkaline range of $\mathrm{pH}$ must be provided from 6.5-8.5 which avoids the erosion of the skin at the acid end and irritation of the skin and mucous membranes at the basic end (Sim et al., 2015; Siyang and Kerdcharoen, 2016; Wiranto et al., 2015).

In our country, there is no $\mathrm{pH}$ monitoring at defined frequencies or average data published on publicly accessible websites. Therefore, the Environmental Protection Agency (EPA) is concerned because of the growing fear of polluted water supplies by criminal industrial and environmental incursions (Taufiqurrahman et al., 2016; Oscanoa et al., 2016; Murphy et al., 2015).
The increased risk of water disruption in the local and global levels is likely to occur more frequently than is happening today. This proposed method will effectively help integrate technology into growing wireless sensor networks which would allow rapid collation, detection, monitoring of pollution and pollution site identification (Marais et al., 2016; Kafli et al., 2016; Haider et al., 2016).

Implementation: This system uses three sensors (turbidity, temperature and $\mathrm{pH}$ ) and the Arduino controller connected to the internet of things, the microcontroller processing module and the GSM transmission module. The three sensors acquire data in the analogy signals by using the $\mathrm{ADC}$ converter that converts the three information signals into digital format. The digital signals are passed to the Arduino controller which is together with the transmission module. The microcontroller of Arduino examine itself and deal with digital information, the GSM Model sends the water quality factors to the smartphone through SMS which can be viewed on the LCD screen.

\section{MATERIALS AND METHODS}

Proposed system: The proposed method is used to overcome defects in existing methods. Arduino Board $(\mathrm{AB})$ was adopted as a basic controller for various sensors to control water quality. Figure 1 shows the water quality monitoring system. The Arduino microcontroller

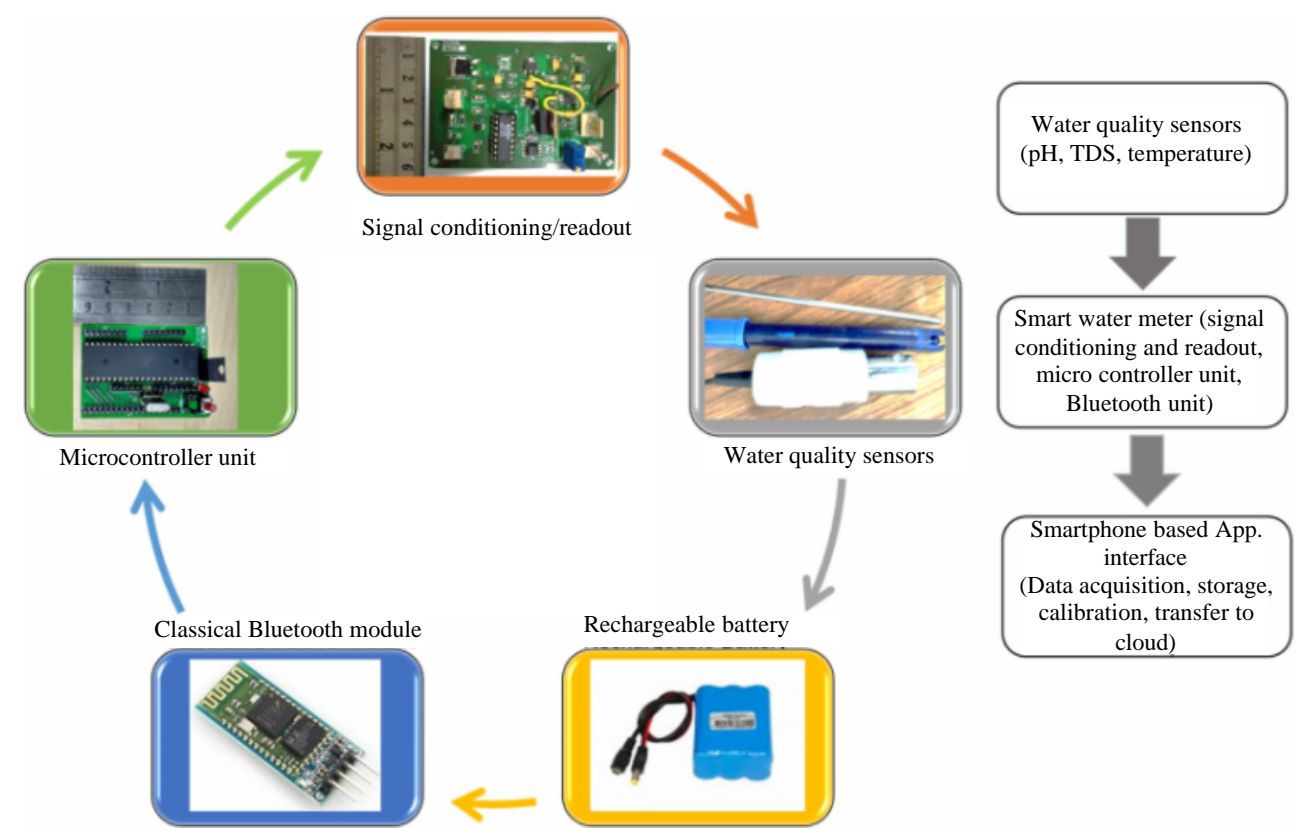

Fig. 1: a) Diagram of intelligent water meter prototypes and various components and b) Integration scheme of smart WQ 
accepts the information and processes the information gathered by the sensors through the GSM module. The analysis of data is processed by the microcontroller in the Arduino module and transmitted to the central server via. the GSM/Wi-Fi module using the data communication module. Collection of generous data will be displayed in real time. The Arduino microcontroller is based on real-time support for embedded tracking and simulation. It is considered to be the main requirement for extended application control for consumer's access and consumes low power.

Internet of Things (IoT): The basic concepts behind the technology of the internet of Things (IoT) are implemented in that the device integrates with the virtual world of the Internet and interacts with it by tracking and monitoring the sensor of the object and its environment. For this reason, the global network of smart devices that can sense and interact with their environment using the internet to connect to and interact with users and other systems (Da Silva Junior et al., 2016; Cloete et al., 2016; Ranjbar and Abdalla, 2017; Robert et al., 2016).

ThingSpeak API: ThingSpeak is an open source application for the Internet of Things (IoT) Application Programming Interface (API) to store and retrieve data from things using HTTP protocol over the internet or over a local network. It allows ThingSpeak to create applications to register the sensor data and the applications to track the locations and the social network of things with status updates (Santos et al., 2016; Chandrappa et al., 2017).

Hardware components (Sowmya et al., 2017; Das and Jain, 2017) pH sensor: The $\mathrm{pH}$ sensor (SKU: SEN0161) is a sensor to detect the value of the $\mathrm{pH}$ of the water. The term " $\mathrm{pH}$ " starts from the Latin and is an abbreviation for "potential hydrogen "or" power of hydrogen". The $\mathrm{pH}$ is the concentration of hydrogen ion in aqueous solutions indicating the acidity and alkalinity in a solution.

A measure of the $\mathrm{pH}$ is a logarithmic scale that ranges from $0-14$ and a neutral point is 7 . The normal range of $\mathrm{pH}$ is 6-8.5. A value $>7$ is for an alkaline solution and values below 7 are indications of the acidic solution as shown in Fig. 2.

TDS sensor: The dissolved solids are all the minerals, salts, metals that are dissolved in the water. The Total Dissolved Solids (TDS) include organic salts (primarily calcium, magnesium, potassium, sodium, bicarbonates, chlorides and sulfates) and some small amounts of organic matter dissolved in water. The TDS sensor kit is compatible with IoT devices when plugged and played

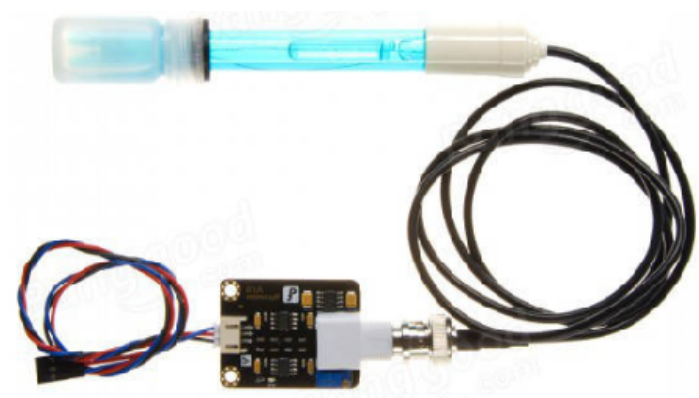

Fig. 2: $\mathrm{pH}$ sensor

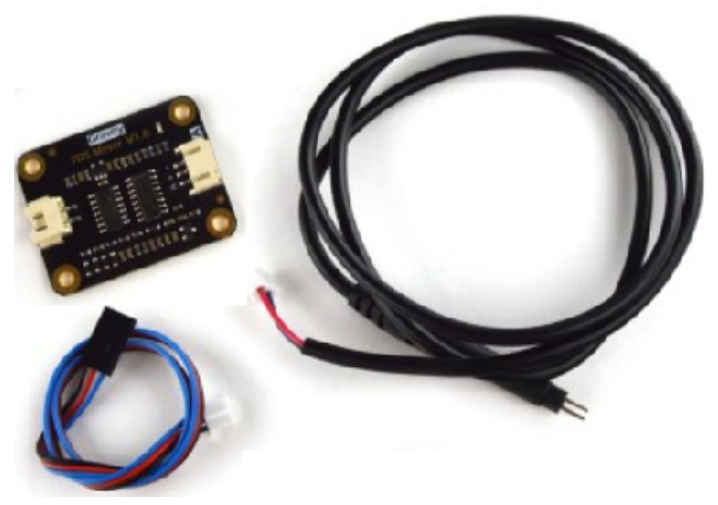

Fig. 3: TDS sensor

and can easily be used to build a detector of TDS measuring the value of TDS of the liquid as shown in Fig. 3. The sensor supports $3.3 \sim 5.5 \mathrm{~V}$ wide voltage input and $0 \sim 2.3 \mathrm{~V}$ analog voltage output, making it compatible with 5 or $3.3 \mathrm{~V}$ control system or circuit board. The measurement range of the TDS sensor is $0 \sim 1000$ ppm.

Temperature sensor: The temperature sensor (DS18B20) was used to measure the temperature of the water. When a precise measurement is required, you should always look at the temperature. An increase in the temperature of the water increases the rate of ionization. Changes in the value of $\mathrm{pH}$ and turbidity vary with temperature. The $\mathrm{pH}$ is related to the temperature and as temperature increases, the ionization rate increases and vice versa. The temperature plays a crucial role in the measurement of the quality of the water.

The temperature is a key factor to determine other applications for the analysis of the quality of the water. We used the DS18B20 to measure temperatures between -55 and $125^{\circ} \mathrm{C}$. This temperature probe digital sealed accurately measures temperature in a humid environment as shown in Fig. 4. 


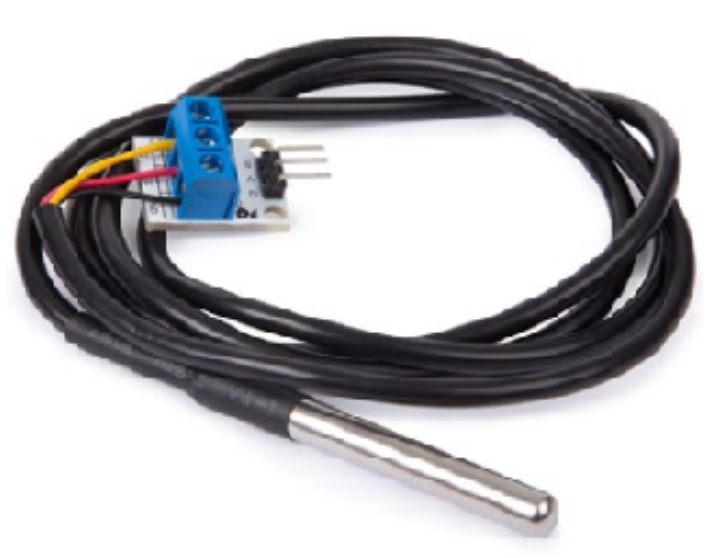

Fig. 4: Temperature sensor

(a)

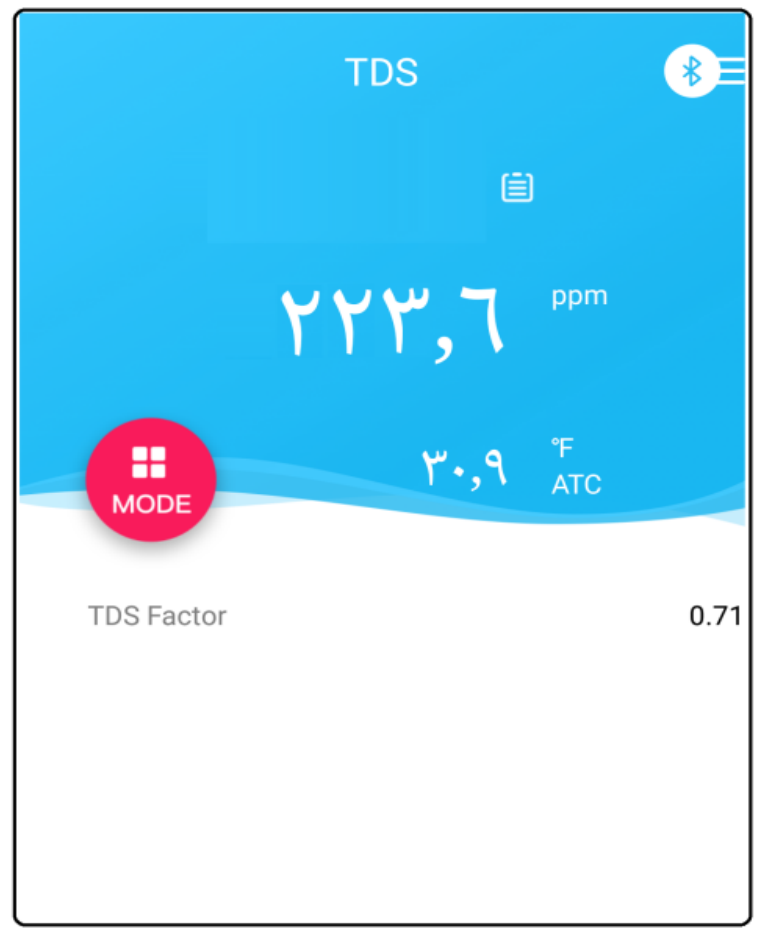

\section{RESULTS AND DISCUSSION}

The system uses three sensors: A temperature sensor (DS18B20), a pH sensor (SKUSEN0161) and a turbidity Sensor (SEN0244). Temperature and turbidity sensors were directly connected to the microprocessor. The $\mathrm{pH}$ probe had a very high resistance and was also susceptible to noise. Then our goal was to select an op-amplifier (TL072) that would be sufficient to prevent the probe from loading and a buffer circuit separated the probe's high impedance from our low impedance of microprocessors (Fig. 5 and Table 1).

Temperature and turbidity sensors provided digital input connected to Arduino digital contacts. The $\mathrm{pH}$ probe connected to the $\mathrm{pH}$ circuit provided an analog

(b)

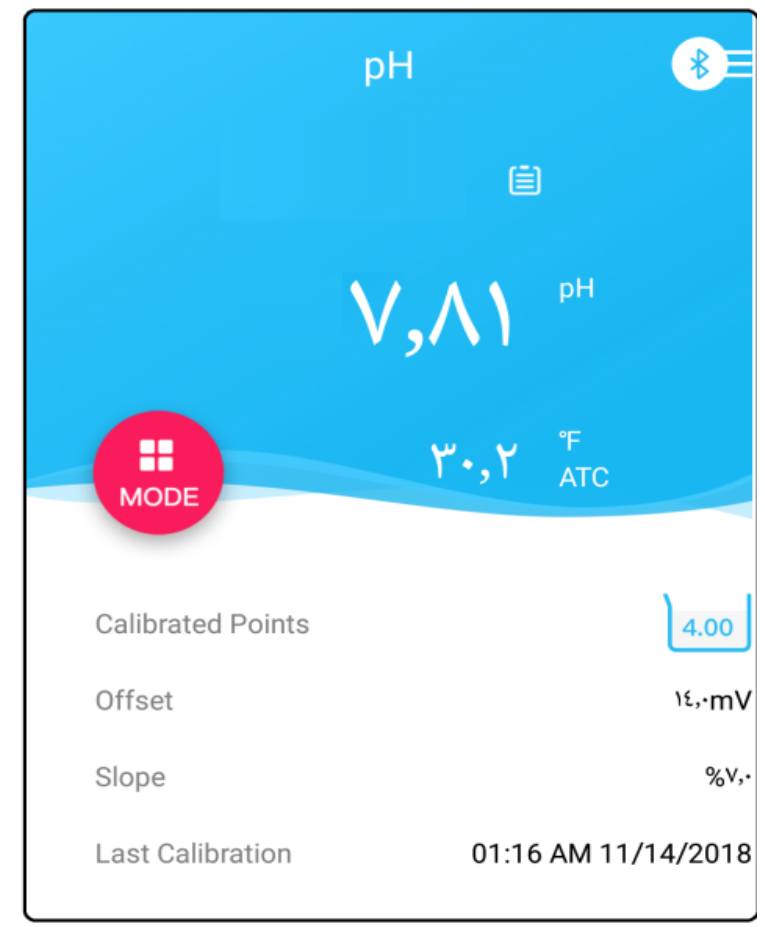

Fig. 5: Desktop application showing real-time: a) TDS and temperature readings and b) $\mathrm{pH}$ and temperature readings of River Shatt Al-Arab

Table 1: Summary of the physical / chemical characteristic of the Shatt Al Arab River water and standard value per WHO requirements

\begin{tabular}{|c|c|c|c|c|c|}
\hline$\underline{\text { Parameters }}$ & Minimum & Maximum & Average & $\mathrm{SD}$ & Standard permissible value (Sn) \\
\hline pH & 7.1 & 8.1 & 7.634 & 0.214 & 8.5 \\
\hline $\mathrm{Ca}\left(\mathrm{mg} \cdot \mathrm{L}^{-1}\right)$ & 144.3 & 504.0 & 289.061 & 111.676 & 75 \\
\hline $\mathrm{Mg}\left(\mathrm{mg} \cdot \mathrm{L}^{-1}\right)$ & 240.3 & 863.0 & 463.645 & 153.422 & 50 \\
\hline Total hardness (mg. $\mathrm{L}^{-1}$ ) & 381.0 & 1455.5 & 763.095 & 248.139 & 500 \\
\hline $\mathrm{SO}^{4}\left(\mathrm{mg} \cdot \mathrm{L}^{-1}\right)$ & 191.0 & 994.5 & 451.248 & 172.062 & 250 \\
\hline $\mathrm{Cl}\left(\mathrm{mg} . \mathrm{L}^{-1}\right)$ & 427.1 & 1498.7 & 839.481 & 254.188 & 200 \\
\hline TDS (mg.L $\left.\mathrm{L}^{-1}\right)$ & 1060.0 & 3670.0 & 2062.139 & 651.988 & 500 \\
\hline $\mathrm{EC}(\mu \mathrm{sec} / \mathrm{cm})$ & 2016.1 & 11723.3 & 5602.160 & 2298.326 & 1000 \\
\hline
\end{tabular}


output, so, it is that was connected to the analog pins of Arduino Uno. All data collected were wirelessly transmitted to smartphones. In order to facilitate this transmission, we used the receiver of the $\mathrm{WiFi}$ transmitter that was connected to Arduino.

To test the preparation of the environment, the prototype was tested and the temperature was displayed in terms of the degree of temperature, turbidity in terms of volts and $\mathrm{pH}$ in terms of $\mathrm{pH}$ level. This data was downloaded wirelessly and displayed in terms of graphics as shown in Fig. 5 and Table 1.

\section{CONCLUSION}

Internet of Things (IoT) and its services have become part of our daily lives forms of work and business. IoT is changing the future of technologies through which one can access any type of information. A real-time water quality monitoring system was tested in real-time, efficiency and inexpensiveness. The level of contamination in the bodies of water was governed and sudden warnings were sent to the public through messages and alarms. This system could provide the necessary warning and protection about the diseases caused by the presence of metals and contaminants in water. So that, severity level of pollutants in Basra's rivers can be taken immediately. The monitoring task can be done using less trained people and the installation of the system can be done easily when it is close to the destination area.

\section{RECOMMENDATIONS}

\section{Advantages:}

- Automatic operation will reduce the time to measure the parameters

- Low maintenance costs

- Prevention of water diseases

In general, the quality of the water of the River Shatt Al Arab is not in good condition for the purpose of drinking and showing all the chemical agents considered that the quality of the water in all the control stations is unsuitable for consumption due to the high levels of concentration of pollutants. It showed that most of these contaminants is in a growing trend which means an increase in the focus levels. Further studies, are needed to study the origin of contaminants in order to avoid any deterioration in the water quality of the Shatt Al-Arab River. In the year under study, it showed higher levels of focus on most of the chemical parameters. The values calculated on water during the study period are found not valid which means that the water quality is not good and not suitable for drinking and other uses.

\section{ACKNOWLEDGEMENTS}

The researcher would like to thank the editor and reviewers for their constructive comments and suggestions on this study. This research was supported by the Southern Technical University/Basrah, (BETC) and the Ministry of Higher Education and Scientific Research/(No.02122018). I would like to express my deep gratitude to the Ms. Janan Abd Ali Al-Hajji and Dr. Shaorong Wang.

\section{REFERENCES}

Amruta, M.K. and M.T. Satish, 2013. Solar powered water quality monitoring system using wireless sensor network. Proceedings of the 2013 International Mutli-Conference on Automation, Computing, Communication, Control and Compressed Sensing (iMac4s), March 22-23, 2013, IEEE, Kottayam, India, ISBN:978-1-4673-5089-1, pp: 281-285.

Anonymous, 2019. Understand your things. MathWorks, Natick, Massachusetts, USA. https:/thingspeak.com/

Anuradha, T., Bhakti, R. Chaitra and D. Pooja, 2018. IoT based low cost system for monitoring of water quality in real time. Intl. Res. J. Eng. Technol., 5: 1658-1663.

Barabde, M.N. and S.R. Danve, 2015. Continuous water quality monitoring system for water resources at remote places. Intl. J. Eng. Res. Gen. Sci., 3: 172-177.

Bhardwaj, R.M., 2011. Overview of Ganga River pollution. Central Pollution Control Board, Delhi. https://cdn.cseindia.org/userfiles/ganga_pollution_ raj.pdf

Chandrappa, S., L. Dharmanna, S.S.U.V. Bhatta, C.M. Sudeeksha and M.N. Suraksha et al., 2017. Design and development of IoT device to measure quality of water. Intl. J. Mod. Educ. Comput. Sci., 9: 50-56.

Cloete, N.A., R. Malekian and L. Nair, 2016. Design of smart sensors for real-time water quality monitoring. IEEE. Access, 4: 3975-3990.

Da Silva Junior, A.G., S.T. De Lima Sa, D.H. Dos Santos, A.P.F. De Negreiros and J.M.V.B. De Souza Silva et al., 2016. Towards a real-time embedded system for water monitoring installed in a robotic sailboat. Sens., 16: 1-19.

Das, B. and P.C. Jain, 2017. Real-time water quality monitoring system using Internet of things. Proceedings of the 2017 International Conference on Computer, Communications and Electronics (Comptelix), July 1-2, 2017, IEEE, Jaipur, India, ISBN:978-1-5090-4709-3, pp: 78-82. 
Haider, A., A.B. Roy, R. Sharma, V.N. Hegde and S. Kumuda, 2016. AquaPredicto-Freshwater quality management system for lakes. Proceedings of the 2016 IEEE International Conference on Region 10 Humanitarian Technology (R10-HTC), December 21 23, 2016, EEE, Agra, India, ISBN:978-1-5090-4178-7, pp: $1--8$.

Kafli, N., M.Z. Othman and K. Isa, 2016. Unsupervised floating platform for environmental monitoring. Proceedings of the 2016 IEEE International Conference on Automatic Control and Intelligent Systems (I2CACIS), October 22, 2016, IEEE, Selangor, Malaysia, ISBN:978-1-5090-4187-9, pp: 84-89.

Le Dinh, T., W. Hu, P. Sikka, P. Corke and L. Overs et al., 2007. Design and deployment of a remote robust sensor network: Experiences from an outdoor water quality monitoring network. Proceedings of the 32nd IEEE International Conference on Local Computer Networks (LCN 2007), October 15-18, 2007, IEEE, Dublin,Ireland,ISBN:978-0-7695-3000-0, pp: 799-806.

Marais, J.M., D.V. Bhatt, G.P. Hancke and T.D. Ramotsoela, 2016. A web-based swimming pool information and management system. Proceedings of the 2016 IEEE 14th International Conference on Industrial Informatics (INDIN), July 19-21, 2016, IEEE, Poitiers, France, ISBN:978-1-5090-2871-9, pp: 980-985.

Murphy, K., B. Heery, T. Sullivan, D. Zhang and L. Paludetti et al., 2015. A low-cost autonomous optical sensor for water quality monitoring. Talanta, 132: 520527.

Oscanoa, H., H. La Chira and F. Cuellar, 2016. Design and development of a Station for remote monitoring of non-domestic wastewater quality. Proceedings of the 2016 EEEE International Conference on ANDESCON, October 19-21, 2016, IEEE, Arequipa, Peru, ISBN:9781-5090-2533-6, pp: 1-4.

Papageorgiou, P., 2003. Literature survey on wireless sensor networks. Master Thesis, University of Maryland, College Park, Maryland.

Pavana, N.R. and M.C. Padma, 2016. Design of low cost system for real time monitoring of water quality parameters in IOT environment. Intl. J. Adv. Res. Comput. Sci. Manage. Stud., 4: 12-15.

Qiao, T.Z. and L. Song, 2010. The design of multiparameter online monitoring system of water quality based on GPRS. Proceedings of the 2010 International Conference on Multimedia Technology, October 2931, 2010, IEEE, Ningbo, China, ISBN:978-1-4244-78712, pp: 1-3.

Ranjbar, R. and A.H. Abdalla, 2017. Development of an autonomous remote access water quality monitoring system. Indonesian J. Electr. Eng. Comput. Sci., 8: 467-474.
Robert, E., M. Grippa, L. Kergoat, S. Pinet and L. Gal et al., 2016. Monitoring water turbidity and surface suspended sediment concentration of the Bagre Reservoir (Burkina Faso) using MODIS and field reflectance data. Intl. J. Appl. Earth Obs. Geoinf., 52: 243-251.

Santos, D., A.G. Silva Junior, A. Negreiros, J.V. Boas and J. Alvarez et al., 2016. Design and implementation of a control system for a sailboat robot. Rob., 5: 1-24.

Sim, S.F., T.Y. Ling, S. Lau and M.z. Jaafar, 2015. A novel computer-aided multivariate water quality index. Environ. Monit. Assess., 187: 1-11.

Siyang, S. and T. Kerdcharoen, 2016. Development of unmanned surface vehicle for smart water quality inspector. Proceedings of the 2016 13th International Conference on Electrical Engineering/Electronics, Computer, Telecommunications and Information Technology (ECTI-CON), June 28- July 1, 2016, EEE, Chiang Mai, Thailand, ISBN: 978-1-4673-9750-6, pp: 15.

Sowmya, C., C.D. Naidu, R.P. Somineni and D.R. Reddy, 2017. Implementation of wireless sensor network for real time overhead tank water quality monitoring. Proceedings of the 2017 IEEE 7th International Conference on Advance Computing (IACC), January 5-7, 2017, EEE, Hyderabad, India, ISBN:978-1-50901561-0, pp: 546-551.

Taufiqurrahman, N. Tamami, D.A. Putra and T. Harsono, 2016. Smart sensor device for detection of water quality as anticipation of disaster environment pollution. Proceedings of the 2016 International Symposium on Electronics (IES), September 29-30, 2016, IEEE, Denpasar, Indonesia, ISBN:978-1-50901641-9, pp: 87-92.

Wang, F., L. Hu, J. Zhou and K. Zhao, 2015. A survey from the perspective of evolutionary process in the internet of things. Intl. J. Distrib. Sens. Netw., 11: $1-9$.

Wiranto, G., Y.Y. Maulana, I.D.P. Hermida, I. Syamsu and D. Mahmudin, 2015. Integrated online water quality monitoring. Proceedings of the 2015 International Conference on Smart Sensors and Application (ICSSA), May 26-28, 2015, IEEE, Kuala Lumpur, Malaysia, ISBN:978-1-4799-7364-4, pp: 111-115.

Ye, W., J. Heidemann and D. Estrin, 2002. An energyefficient MAC protocol for wireless sensor networks. Proceedings of the 21st Annual International Joint Conference on the IEEE Computer and Communications Societies Vol. 3, June 23-27, 2002, IEEE, New York, USA., ISBN:0-7803-7476-2, pp: 1567-1576. 